\title{
Erratum
}

\section{Inflation from the superstring vacuum}

\author{
[Int. J. Mod. Phys. A, Vol. 24, Nos. 20 \& 21 (2009) 4021-4037] \\ M. D. Pollock \\ V. A. Steklov Mathematical Institute, Russian Academy of Sciences, \\ Ulitsa Gubkina 8, Moscow 119991, Russia* \\ mdp30@cam.ac.uk
}

Received 22 September 2016

Published 30 September 2016

The following are corrections to errors in the published paper:

(1) On page 4021 , in line 10 , printed as " $\kappa_{0}^{-2}\left[1+15 \zeta(3) \bar{\chi} / 16 \lambda B_{\mathrm{r}}^{3}+\bar{\chi} / 384 \pi^{2} A_{\mathrm{r}}\right]$ " should read " $\kappa_{0}^{-2}\left[1-15 \zeta(3) \bar{\chi} / 16 \lambda B_{\mathrm{r}}^{3}-\bar{\chi} / 384 \pi^{2} A_{\mathrm{r}}\right]$ "

(2) On page 4021, in line 12, printed as "negative" should read "positive or negative"

(3) On page 4021, in line 13, printed as "produce" should read "can produce"

(4) On page 4021 , in line 14 , printed as " $B_{\mathrm{r}}^{(\mathrm{c})}=[15 \zeta(3)|\bar{\chi}| / 16 \lambda]^{1 / 3}$ " should read " $B_{\mathrm{r}}^{(\mathrm{c})}=[15 \zeta(3) \bar{\chi} / 16 \lambda]^{1 / 3 "}$

(5) On page 4021, in line 15 , printed as " $\bar{\chi}=-6$ " should read " $\bar{\chi}=6 "$

(6) On page 4021 , in line 18 , printed as " $V\left(B_{\mathrm{r}}\right)=\frac{15}{32} \zeta(3) \bar{\chi} \lambda^{-1} \kappa_{0}^{-2} R B_{\mathrm{r}}^{-3}-\ldots$ " should read " $V\left(B_{\mathrm{r}}\right)=-\frac{15}{32} \zeta(3) \bar{\chi} \lambda^{-1} \kappa_{0}^{-2} R B_{\mathrm{r}}^{-3}-\ldots$ "

(7) On page 4021 , in line 24 , printed as " $V\left(A_{\mathrm{r}}\right)=\bar{\chi} \kappa_{0}^{-2} R A_{\mathrm{r}}^{-1} / 768 \pi^{2}-\ldots$ " should read " $V\left(A_{\mathrm{r}}\right)=-\bar{\chi} \kappa_{0}^{-2} R A_{\mathrm{r}}^{-1} / 768 \pi^{2}-\ldots$ "

\footnotetext{
* Temporary address.
} 
(8) On page 4023, Eq. (8) should read

$$
\kappa^{-2}=\kappa_{0}^{-2}\left[1-\frac{15 \zeta(3) \bar{\chi}}{16 \lambda B_{\mathrm{r}}^{3}}-\frac{\bar{\chi}}{384 \pi^{2} A_{\mathrm{r}}}\right] .
$$

(9) On page 4023 , in line 12 , printed as " $\bar{\chi}=-6$ " should read " $\bar{\chi}= \pm 6$ "

(10) On page 4023, in line 19, Eq. (10), printed as "= $1.69 \times \ldots$.. should read "=干1.69 ×.."

(11) On page 4023, in line 20, Eq. (10), printed as "= $7.92 \times \ldots "$ should read "= $77.92 \times \ldots "$

(12) On page 4024, in line 11, printed as "Eqs. (10)," should read "Eqs. (10), setting $\bar{\chi}=6$ and"

(13) On page 4024, Eq. (15) should read

$$
B_{\mathrm{r}}^{(\mathrm{c})}=\left[\frac{15 \zeta(3) \bar{\chi}}{16 \lambda}\right]^{1 / 3}=0.357\left(\frac{15 \pi^{2}}{\lambda}\right)^{1 / 3}\left(\frac{\bar{\chi}}{6}\right)^{1 / 3} .
$$

(14) On page 4024, in line 16, printed as "Minkowski space and anti-" should read "Minkowski space and"

(15) On page 4024, in line 17, printed as "negative" should read "positive"

(16) On page 4024, Eq. (16) should read

$$
\Lambda_{\mathcal{R}^{4}}=\left[\frac{18}{337 \zeta(3)+1 / 2}\right]^{1 / 3} A_{\mathrm{r}}^{-1} \kappa^{-2 / 3} \kappa_{0}^{-4 / 3} .
$$

(17) On page 4025, Eq. (19) should read

$$
K \equiv\left|\frac{\Lambda_{\mathcal{R}^{2(\text { anom })}}}{\Lambda_{\mathcal{R}^{4}}}\right|=8640 \pi^{2}\left[\frac{337 \zeta(3)+1 / 2}{18}\right]^{1 / 3} \frac{A_{\mathrm{r}}}{k_{2}}\left|\frac{\kappa_{0}}{\kappa}\right|^{4 / 3} .
$$

(18) On page 4025 , in line 10 , printed as " $\Lambda_{\mathcal{R}^{4}}<0$ " should read " $\Lambda_{\mathcal{R}^{4}}>0$ "

(19) On page 4025, in line 11, printed as "anti-de Sitter" should read "de Sitter"

(20) On page 4025, Eq. (23) should read

$$
V\left(B_{\mathrm{r}}\right)=-\frac{15}{32} \zeta(3) \bar{\chi} \lambda^{-1} \kappa_{0}^{-2} R B_{\mathrm{r}}^{-3}-A_{\mathrm{r}} \tilde{B} \mathcal{R}^{2} B_{\mathrm{r}}^{-2} .
$$

(21) On page 4026, in line 3, printed as "Since $\bar{\chi}<0,{ }^{27}$ note first that" should read "Mirror Calabi-Yau manifolds exist for which $\bar{\chi}>0$ or $\bar{\chi}<0^{27}$ [see P. Candelas, M. Lynker and R. Schimmrigk, Nucl. Phys. B 341, 383 (1990)]. Note, however, that"

(22) On page 4026, in line 4, printed as "provided that $R<0$, and thus only for a de Sitter" should read "when $R<0[R>0]$, and thus for a de Sitter"

(23) On page 4026, in line 5, printed as "(rather than anti-de Sitter) maximally symmetric space," should read "[anti-de Sitter] maximally symmetric space, only if $\bar{\chi}>0[\bar{\chi}<0], "$ 
(24) On page 4026, line 17 should read

Setting $\bar{\chi}=6$, the constants $V_{1}$ and $V_{2}$ are defined as

(25) On page 4027 , in line 6 , printed as " $R<0$, corresponding to de Sitter space," should read " $R>0$, corresponding to anti-de Sitter space,"

(26) On page 4027, Eq. (36) should read

$$
R=-4 \Lambda_{\mathcal{R}^{4}}=4\left[\frac{18}{337 \zeta(3)+1 / 2}\right]^{1 / 3} A_{\mathrm{r}}^{-1} \kappa_{0}^{-2}\left(\beta^{-3}-1\right)^{1 / 3}
$$

(27) On page 4027, line 11 should read

where $V_{1}^{\prime}=-2\{18 /[337 \zeta(3)+1 / 2]\}^{1 / 3} A_{\mathrm{r}}^{-1} \kappa_{0}^{-4}$ and

$V_{2}^{\prime}=12\{18 /[337 \zeta(3)+1 / 2]\}^{2 / 3} A_{\mathrm{r}}^{-1} \widetilde{B} B_{\mathrm{r}}^{(\mathrm{c})-2} \kappa_{0}^{-4}$.

(28) On page 4027, line 12 should read

The extrema of expression (37) are given by

(29) On page 4027, lines 15-18 should read

which, however, yields no solution when $\left(\beta^{-3}-1\right)^{1 / 3}>0$.

(30) On page 4028, Eq. (42) should read

$$
V\left(A_{\mathrm{r}}\right)=-\frac{1}{768 \pi^{2}} \bar{\chi} \kappa_{0}^{-2} R A_{\mathrm{r}}^{-1}-\left(\frac{1}{4} \mathcal{R}_{\mathrm{E}}^{2}+\widetilde{B} B_{\mathrm{r}}^{-2} \mathcal{R}^{2}\right) A_{\mathrm{r}} .
$$

(31) On page 4028, Eq. (46) should read

$$
V\left(A_{\mathrm{r}}\right) \approx \frac{1}{768 \pi^{2}} \bar{\chi} \kappa_{0}^{-2}|R| A_{\mathrm{r}}^{-1}
$$

(32) On page 4029, Eq. (55) should read

$$
\left.L_{\zeta(3)}^{(4)}\right|_{\text {tree-level }}=\left[\frac{337 \zeta(3)+1 / 2}{288}\right] \alpha^{\prime 2} \Lambda_{\mathcal{R}^{4}}^{4} A_{\mathrm{r}}^{(\mathrm{c}) 3} \alpha_{\mathrm{r}}^{3},
$$

(33) On page 4029, Eq. (56) should read

$$
\Lambda_{\mathcal{R}^{4}}=\left[\frac{18}{337 \zeta(3)+1 / 2}\right]^{1 / 3} A_{\mathrm{r}}^{-1} \kappa_{0}^{-2}\left(1-\alpha_{\mathrm{r}}^{-1}\right)^{1 / 3},
$$

(34) On page 4030, Eq. (61) should read

$$
V_{1}^{\prime \prime}=-64 \pi^{2}\left[\frac{18}{337 \zeta(3)+1 / 2}\right]^{1 / 3} \kappa_{0}^{-4} .
$$

(35) On page 4030, in line 15, printed as " $\partial^{2} V / \partial \alpha_{\mathrm{r}}^{2}<0$ " should read " $\partial^{2} V / \partial \alpha_{\mathrm{r}}^{2}>0 "$

(36) On page 4030 , in line 16 , printed as " $V\left(A_{\mathrm{r}} \rightarrow 0_{+}\right) \rightarrow \infty$ " should read "V $\left(A_{\mathrm{r}} \rightarrow 0_{+}\right) \rightarrow-\infty "$

(37) On page 4030, in line 16, printed as "minima" should read "maxima"

(38) On page 4030, in line 17, printed as "maximum" should read "minimum" 
(39) On page 4030, in Eq. (65), printed as " $V\left(\alpha_{\mathrm{r}}^{(+)}\right)=\cdots>0$ " should read " $V\left(\alpha_{\mathrm{r}}^{(+)}\right)=\cdots<0 "$

(40) On page 4034, in line 2, printed as "anti-de Sitter" should read "de Sitter"

(41) On page 4036, Refs. 10-14, 25 and 28 should read

10. M. D. Pollock, Phys. Lett. B 411, 68 (1997); B 743, 542(C) (2015).

11. M. D. Pollock, Int. J. Mod. Phys. A 16, 3217 (2001); A 27, 1292008(E) (2012); A 31, 1692001(E) (2016).

12. M. D. Pollock, Int. J. Mod. Phys. A 7, 4149 (1992); A 27, 1292005(E) (2012).

13. M. D. Pollock, Phys. Lett. B 436, 275 (1998); B 744, 414(C) (2015).

14. M. D. Pollock, Int. J. Mod. Phys. D 16, 591 (2007); D 23, 1492001(E) (2014).

25. M. D. Pollock, Phys. Lett. B 495, 401 (2000); B 750, 667(C) (2015).

28. M. D. Pollock, Int. J. Mod. Phys. D 13, 923 (2004); D 25, 1692001(E) (2016).

(42) On page 4037, Ref. 33 should read

33. M. D. Pollock, Int. J. Mod. Phys. D 15, 845 (2006); D 22, 1392001(E) (2013). 